\title{
Non-Support for Trainability of Teammate Recognition Based on Movement Perception?
}

\section{KYLIE A. STEEL ${ }^{1}$}

1 Western Sydney University, Penrith, Australia.

Correspondence to: Kylie A. Steel, School of Science and Health, Western Sydney University, The MARCS Institute for Brain Behaviour and Development, Locked Bag 1797, Penrith, NSW, 2751, AUSTRALIA.

email: k.steel@westernsydney.edu.au

https://doi.org/10.20338/bjmb.v13i1.120

HIGHLIGHTS

- Video as a training method did not improve teammate recognition in a perceptual training task.

- Representative design is critical for perceptual learning.

- Recognizing teammates by their swimming gait may not be susceptible to training for experienced players.

$\begin{array}{ll}\text { ABBREVIATIONS } \\ \text { AUC } & \text { Area Under the Curve } \\ \text { nTM } & \text { not teammate } \\ \text { ROC } & \text { receiver operating characteristic } \\ \text { STPa } & \begin{array}{l}\text { superior-temporal- } \\ \end{array} \\ \text { polysensoryarea } \\ \text { TM } & \text { teammate }\end{array}$

PUBLICATION DATA

Received 18122018

Accepted 25042019

Published 01052019
BACKGROUND: Accurate decisions are essential for successful performance in visually and temporally constrained sports environments such as water-polo. Visual cues (uniform and facial) can be obscured by other factors such as water splashes, or partial submersion, thus leading to misclassification of others as teammates and lost scoring affordances. Research suggests that like land gait recognition, swimming gait is also distinguishable from temporally occluded visual stimuli, thus allowing teammate recognition with high accuracy, though not devoid of some errors. To that end investigating the trainability of this perceptual ability has merit as increased recognition accuracy may result in more scoring opportunities.

AIM: Therefore, the purpose of this study was to assess whether teammate recognition accuracy can be enhanced using a video-based training paradigm and, which factors affect this ability.

METHOD: $\mathrm{N}=12$ females (Mean age $=18.75$ years \pm 2.5 ) completed a two-week video training intervention. RESULTS: Statistical analysis demonstrated that participant's performance didn't change significantly after training (kick: $p=0.814$; stroke: $p=0.939$ ), nor was their ability dependent on experience in water-polo (kick: $p=0.141$; stroke: $p=0.169$ ), skill level (kick: $p=0.715$; stroke: $p=0.287$ ), or training adherence $(k i c k ; p=0.536$; stroke $p=0.797$ ). CONCLUSION: While some research suggests that recognition based on biological motion is trainable it was not corroborated in this study and thus requires further investigation as to the mechanisms that contribute to improvement.

\section{INTRODUCTION}

Water-polo is a dynamic invasion sport that requires fast and accurate decision making when passing, in order to take advantage of scoring affordances. ${ }^{1}$ Like any invasion sport that is quick and dynamic (e.g., football, hockey and water-polo), teammate affiliation can be ascertained from uniform or cap design and colour, or facial features. ${ }^{2}$ However, there are instances where these sources of visual information may be obscured by either another player, or the variables created by movement in water (splash or partial submersion). Consequently, the concurrent use of other visual cues is essential for identifying teammates and may include movement signatures, especially those related to locomotion. ${ }^{3}$

A significant body of research has demonstrated that humans are able to recognize numerous features about other individuals based on relative (timing and spatial) information, when viewing degraded visual displays of human motion e.g., point-light displays or video footage (See Steel, Baxter \& Ellem (2015) ${ }^{3}$ for an extensive review). These characteristics include age ${ }^{4}$, deception and emotion ${ }^{5}$, familiars 6 (known individuals), gender ${ }^{7}$, identity 8 , intention and movement type. ${ }^{9}$ Researchers have also determined that humans have

\begin{tabular}{l|l|l|l|l}
\hline Steel & 2019 & VOL.13 & N.1 & https://doi.org/10.20338/bjmb.v13i1.120
\end{tabular}


developed a dedicated neural network sensitive to the dynamics of biological motion that consists of multiple areas of the dorsal pathways (V1-V5/MT), superior-temporalpolysensoryarea, and the superior-temporal-polysensoryarea (STPa). ${ }^{10}$ The evolution of these neural regions have been a result of the need to survive which is aided by the accurate perception of biological motion ${ }^{11}$.

For example, the capacity to recognize an individual in the distance from their movement signature, but not by their face, allows you to recall previous interactions that may have been injurious. Therefore, the ability to determine an individual's intention or identity, particularly if the intention of this individual seems unfriendly, enables a person to pre-plan their interactions. By pre-planning a person may therefore avoid the other individual, especially when processed in an automatic manner, alternatively a person can prepare to defend oneself and those close to them.

Despite an extensive body of research related to biological motion that allows the recognition of various characteristics and features (e.g., movement type, gender, and identity), only a limited number of real-world applications for this research have been pursued $^{3}$. These include; biometrics ${ }^{12}$, rehabilitation ${ }^{13}$, safety garments ${ }^{14}$, and sport ${ }^{1}$, with biometrics and safety garment-based research attracting substantial interest within the biological motion domain.

Biometrics is the use of features and characteristics of an individual that are idiosyncratic and specific to that person, for example, finger print, iris pattern, voice recognition and more recently walking and running gait. ${ }^{12}$ The research demonstrates that all individuals have a unique way of walking and running, which conveys movement information allowing person recognition when facial features are obscured, e.g., surveillance footage. ${ }^{12}$ Biological motion research has also proved essential in the development and design of safety or high visibility garments that are used by cyclists, construction workers and traffic controllers. The research has specifically led to the strategic placement of reflective material on joints that are shown to effectively convey human movement in light restricted environments such as night-time or rainy conditions. ${ }^{14}$

Based on the individuality of gait and the ability of observers to recognise others from this perceptual source, Steel, Adams, and Canning ${ }^{15,1}$, proposed that if individuals generally could perform this task when observing restricted visual displays, it is likely that sports people, who rely heavily on information gathered from the visual field, could do so also. The researchers demonstrated that most athletes in team sports were able to achieve this task at a level significantly above chance when observing temporally occluded footage regardless of movement type, e.g., stroke and kick in swimming ${ }^{1}$ and whole-body movements in running ${ }^{15}$, gender ${ }^{15}$, or age..$^{16}$ However some participants did not perform well which is in opposition to other visually-based findings for athletes. ${ }^{17}$ The evidence broadly demonstrates that experts are skilled at using visual information and have superior visual strategies when engaging in sport, ${ }^{17}$ (See Appelbaum and Erickson, (2018) (17 $^{17}$ an extensive review). Despite this some athletes in previous studies conducted by the Steel, Adams, and Canning team, demonstrated inferior perpetual abilities related to teammate recognition based on biological motion. Therefore, the purpose of the current study was to investigate whether a video-based method was a viable option for training the perceptual skill associated with using biological motion cues for person recognition with an emphasis on highly skilled athletes?

\begin{tabular}{l|l|l|l|l}
\hline Steel & 2019 & VOL.13 & N.1 & https://doi.org/10.20338/bjmb.v13i1.120
\end{tabular}




\section{METHODS}

\section{Participants}

Twelve female water-polo players (Mean age $=18.75, \pm 2.5 y e a r s$ ) who competed on average ( $8 \pm 2.12$ years) in a national league team volunteered to participate in this study. $A$ further eighteen high level club swimmers acted as distractors in test footage but did not take part in testing or the training intervention. All participants provided signed consent prior to involvement in the study as required by the approving Humans Ethics Committee.

\section{Stimulus Design and Construction}

Water-polo players and club swimmers were filmed performing a head down freestyle sprint past a fixed forward-facing digital video camera (25fps/Sony HDR FX1000) situated on the pool deck. All swimmers wore the same style and colour of swimwear, cap and goggles. Each individual's clip was then split into two segments, with the first part of the clip consisting of the arm component of the swim stroke, measured from the point the first hand entered the camera view finder and ended when the gluteal region entered view (Figure 1). The same process was used for the second half of the sequence that showed the kick component of the stroke which was measured from the frame where the gluteal region was about to leave the camera view finder and ended only when the feet where no longer in view (Figure 1). This footage was then edited into two separate randomized testing sequences (stroke and kick) using Adobe Premier Pro CS3 software (Adobe Systems Inc; 345 Park Ave, San Jose, CA, USA).

Specifically, each of the two test sequences started with a main title slide (5seconds) followed by a blank slide (3seconds in duration), a 2second clip preparing the observer for the first clip, and then a clip, this was repeated for every clip with the exception of the main title slide which was only seen at the start of the sequence $1,15,16$. Each swimmer was only seen once in each of the two randomized sequences, therefore each sequence contained 30 individual clips. The decision to split stroke and kick clips was chosen, as incorporating the entire swim stroke cycle of each participant was not ecologically valid ${ }^{1}$ in that it was often longer than 1 second. In sport, players must frequently make decisions in less than $500 \mathrm{msec}$ therefore the use of temporal occlusion was important to the objective of the study.
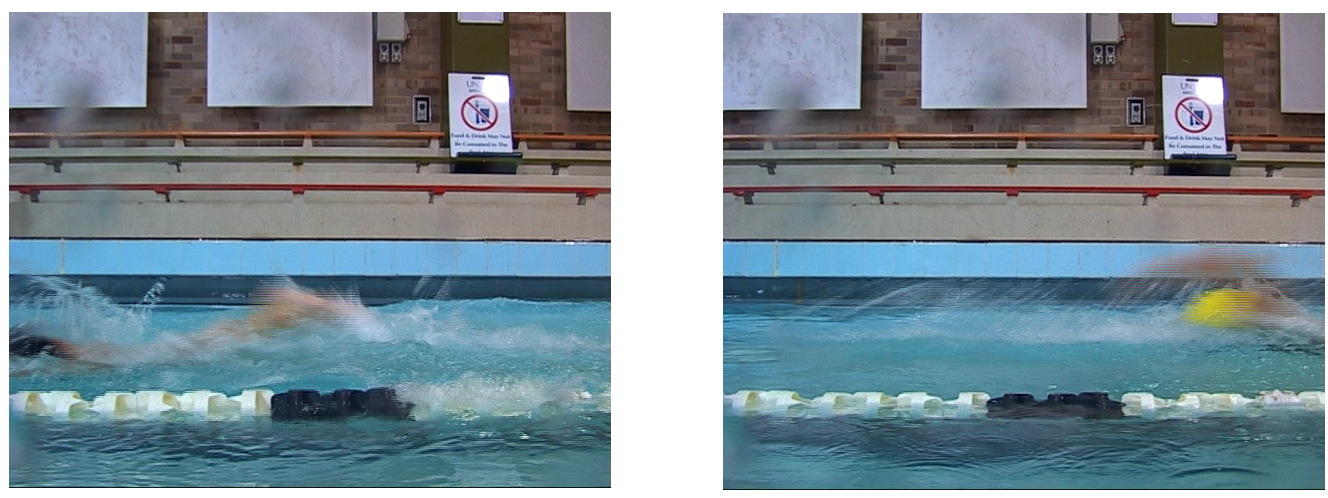

Figure 1. Examples of side view footage used for testing teammate recognition ability.

\begin{tabular}{l|l|l|l|l}
\hline Steel & 2019 & VOL.13 & N.1 & https://doi.org/10.20338/bjmb.v13i1.120
\end{tabular}




\section{Test Procedures}

Each water-polo participant only, was asked to observe the same randomized test sequence at the pre, post (two weeks later) and post 2 (two weeks after the post-test) tests where they would indicate whether the swimmer in each video clip was a teammate or not based on the swimmer's movement style. This was achieved using a pen and paper method where the participant had to indicate, via a check mark, their level of certainty that the swimmer they observed was either not a teammate (nTM) (1-3) compared to 4-6 for level of certainty that the swimmer was a teammate (TM) (Figure 2). Each decision had to be made after one video clip and before the next which was a 5second interval, for every clip in the sequence.

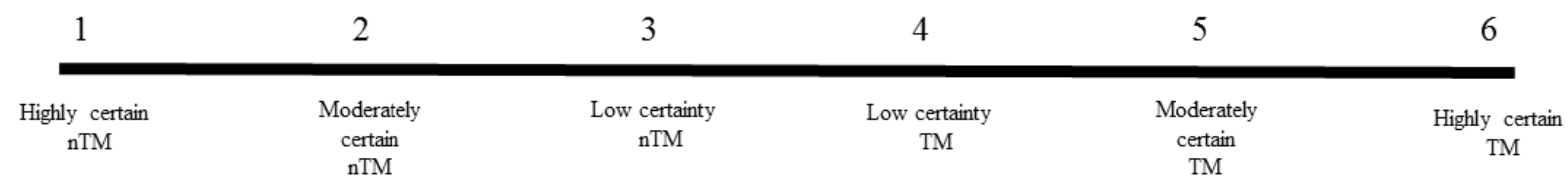

Figure 2. Decision choice confidence scores with 1 being the most certain the swimmer was not a teammate through to 6 which indicated most certain the swimmer was a teammate.

\section{Intervention Procedure}

A separate video sequence was constructed for the intervention period that consisted of a randomized series of twelve individual video clips of the water-polo teammates only, which showed each swimmer performing a swim task. The players were asked to enter the pool and then face toward the camera (25fps/ Sony HDR FX1000) thus indicating to the viewer who they were and that the subsequent swim sequence was theirs (Figure $3 \mathrm{~A}$ ). The player was instructed to swim as fast as they could around two buoys (Figures $3 \mathrm{~B}$ and $3 \mathrm{C}$ ) and back to the start position (Figure 3D). The buoys were placed $5 \mathrm{~m}$ away from the start position at a 45-degree angle to the left and right, thus forming a triangular path (Figure 4). Participants in group one $(\mathrm{N}=6)$ were asked to watch the training sequence (3minutes in duration) each day for fourteen days, therefore fourteen 3minute training sessions, then complete a post-test, no other explicit instructions were given. After an additional two weeks of no training, which acted as a control period, they completed a second post-test. Participants in group two $(\mathrm{N}=6)$ received no training in the first two weeks which acted as a control period, while in weeks three and four they completed the same training intervention as group one which included the viewing of the 3minute training sequence each day for fourteen days.

\begin{tabular}{l|l|l|l|l}
\hline Steel & 2019 & VOL.13 & N.1 & https://doi.org/10.20338/bjmb.v13i1.120
\end{tabular}


Brazilian Journal of Motor Behavior
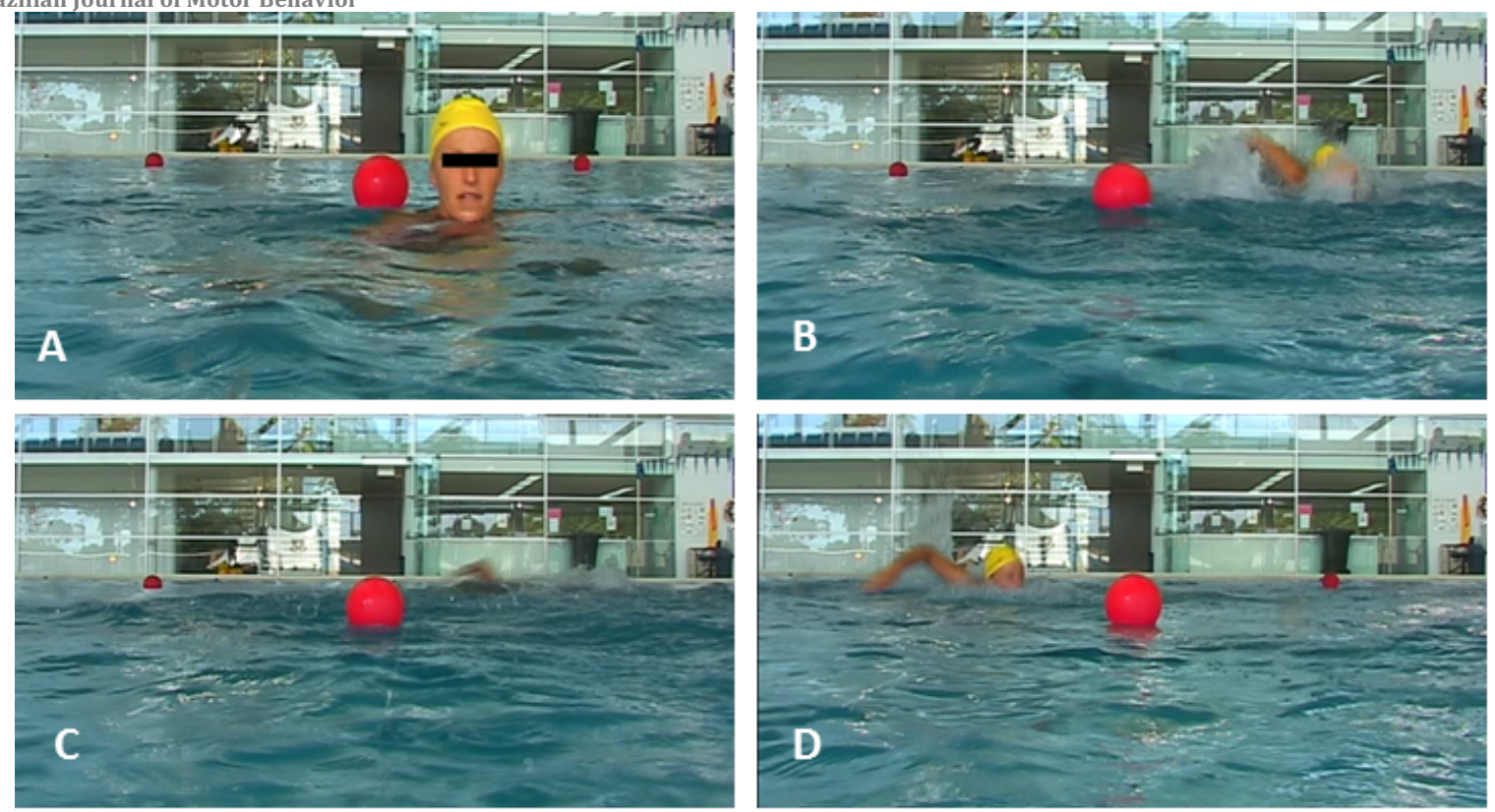

Figure 3. 3A.Shows player identity; 3B. The player swims toward the first buoy; 3C. The players swims between buoys 1 and 2; 3D. Players returns to starting position.

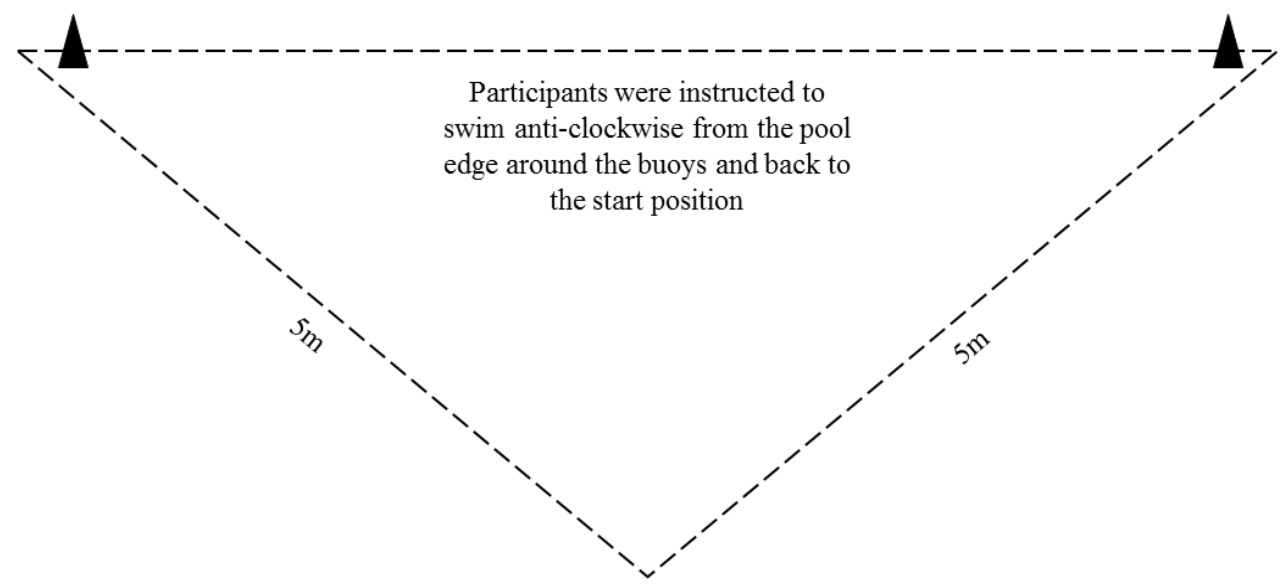

Pool start position

Figure 4. Shows the path travelled by the swimmers in the training sequence.

\section{Data Analyses}

Initial data analysis utilized Signal Detection Theory methods where the teammates were signals and the non-teammates were noise. ${ }^{18}$ Using the receiver operating characteristic (ROC) curve subroutine of SPSS Windows (Version 25; SPSS Inc, Chicago, IL), swimmer status (teammate/non-teammate) was the state variable while the certainty related to decision accuracy and was the continuous variable (1-6). The ROC analysis creates an Area Under the Curve (AUC) whereby the value of 0.5 represents chance while 1.0 represents the highest possible score. The condition for normality was met using a 
Brazilian Journal of Motor Behavior

\section{Research Article}

Shapiro-Wilks test and an analysis of variance was then conducted on the AUC scores to test for significance and was set at $p<0.05$. Further analysis was conducted using a general linear model to determine statistical significance, effect size and F-distribution using Greenhouse-Geisser for tests of within-subject effects in relation to choice accuracy when comparing to players' skill level, training adherence, and playing experience.

\section{RESULTS}

Examination of the AUC generated data demonstrated that while some participants performed the task at a levels significantly above chance, most participants performed at a chance level (Table 1) for each test irrelevant of sequence (stroke or kick), or group (1 or 2).

Table 1 - Teammate discrimination accuracy (AUC) scores.

\section{Stroke Sequence}

\begin{tabular}{|c|c|c|c|c|c|c|}
\hline & Pre-test & Post-test & $\begin{array}{c}\text { Post } \\
\text {-test } 2\end{array}$ & Pre-test & Post-test & $\begin{array}{c}\text { Post } \\
\text {-test } 2\end{array}$ \\
\hline \multirow[t]{6}{*}{ Group 1} & 0.568 & 0.531 & $0.714+$ & 0.378 & $0.500+$ & 0.445 \\
\hline & $0.742^{*}$ & 0.576 & 0.482 & 0.578 & 0.417 & 0.443 \\
\hline & 0.682 & $0.737+^{*}$ & 0.693 & 0.578 & $0.654+$ & 0.539 \\
\hline & $0.839^{*}$ & 0.625 & 0.643 & 0.370 & $0.479+$ & 0.333 \\
\hline & 0.604 & $0.891+^{*}$ & $0.893+^{*}$ & 0.500 & 0.375 & $0.607+$ \\
\hline & 0.714 & 0.664 & $0.771+^{*}$ & 0.549 & 0.542 & $0.578+$ \\
\hline Mean Score & 0.692 & 0.671 & $0.699+$ & 0.492 & $0.495+$ & 0.491 \\
\hline \multirow[t]{6}{*}{ Group 2} & 0.708 & 0.573 & $0.633+$ & 0.391 & $0.630+$ & 0.378 \\
\hline & 0.685 & 0.617 & $0.620+$ & 0.523 & 0.510 & $0.547+$ \\
\hline & 0.445 & 0.424 & $0.641+$ & 0.378 & $0.451+$ & $0.594+$ \\
\hline & 0.680 & $0.740+^{*}$ & $0.799+^{*}$ & 0.620 & 0.576 & 0.482 \\
\hline & 0.638 & 0.560 & 0.466 & 0.484 & $0.513+$ & $0.526+$ \\
\hline & 0.659 & $0.680+$ & 0.586 & 0.542 & 0.513 & 0.461 \\
\hline Mean Score & 0.636 & 0.599 & $0.624+$ & 0.490 & 0.532 & 0.498 \\
\hline
\end{tabular}

Significance measured at $p<0.05\left(^{*}\right)$. Increases in performances are highlighted when flowed by a (+).

Kick data analysis elicited the following values for significance and effect sizes related to skill level $p=0.715, n \rho 2=0.103, F(0.517)$, training adherence $p=0.536, n \rho 2=0.057$, $F(0.607)$, and playing experience $p=0.141, n \rho 2=0.215, F(2.463)$. Stroke data analysis revealed similar trends for all three variables; skill level $p=0.287, \eta p 2=0.237, F(1.394)$, training adherence $p=0.797, n \rho 2=0.016, F(0.159)$, and playing experience $p=0.169, n \rho 2$ $=0.187, F(2.066)$.

\section{DISCUSSION}

\begin{tabular}{l|l|l|l|l}
\hline Steel & 2019 & VOL.13 & N.1 &
\end{tabular}


The purpose of the current study was to investigate the trainability of the perceptual ability to discriminate humans based on movement signatures when using a video-based method. Unlike previous research investigating teammate recognition, ${ }^{1,16}$ where most participants were able to distinguish teammates from non-teammates at levels greater than chance, only a limited number of the participants in the current cohort could replicate this trend. Moreover, performance levels for this ability did not significantly change with training, indeed performance decreased after training for some individuals including the most experienced member of the squad.

Frequent training together would presumably provide crucial experiences for person recognition from motion 8,19 , however this was not evident in the current study where participants had trained together over a longer period and at a higher competitive level (national) compared to participants in earlier studies. Future consideration could be given to engaging non-teammates in this type of research whereby participants observe unknowns during pre-testing, watch footage of a sample of some of these individuals during training sessions, then retest watching all participants again. This allows discrimination without previous exposure to any of the viewed individuals thus reducing possible bias.

Further, it is possible that like Cutting and Kozlowski $(1977)^{8}$, participants in other studies were able to demonstrate higher perceptual ability due to more extensive movement information presented in the video clips. In the current study video stimuli provided limited visual information due to the spatial occlusion of the partially submerged swimmers in the training and test footage, in addition to limited temporal exposure. Thus, the observer may require access to global information for recognition that allows a gestalt of visual information to be processed. 6

Alternatively, the lack of representative design ${ }^{20}$ may have contributed to the current findings. The use of different viewing perspectives between the test and training footage may have been problematic as athletes are perceptually attuned to affordances in the environment. Hence, presenting athletes with low-fidelity camera angles may in part compromise their perceptual expertise. Moreover, the length of each clip meant that the test sequence presented very brief visual clips of the side view only where no swimmer was observed for longer than one second, as opposed to the training footage that provided a significantly longer period for observation. For example, the training sequence video clips showed swimmers for up to 10 seconds in order to provide substantial exposure to movement signatures. Specifically, each individual training clip showed the swimmer was identified by facing the camera first then swimming away from the observer, and across a set course before returning. This allowed the observer to view the stroke from three different angles in addition to associating a face with the sequence from the start.

An additional consideration is that future studies should be designed to included participants from the same sport, e.g., swimming or water-polo, not both as swimming kinematics is likely to be different due to the different demands of the sports. For example, water-polo players often lift their head to view the dynamics of the game environment which is not necessary for swimmers.

While not overtly explicit when compared to other examples of instruction, the statement 'watch the video once a day for fourteen days' draws attention to a more conscious level of processing, and may not be the most constructive strategy given the evidence presented here. For example, athletes may gain more benefit from techniques that 
Brazilian Journal of Motor Behavior

are more implicit such as engaging in physical game scenarios where all participants wear the same costume and cap (or no cap), which can also be filmed and viewed by players in concurrent training game analysis sessions. This approach encourages the players to use a variety of sources of visual information for recognition of team affiliation and in a manner that is secondary to the primary task of improving motor skills. Thus an advantage of this strategy is that information is learned below conscious awareness which decreases its susceptibility to game pressure degradation. ${ }^{21}$

Finally, examination of variables such as adherence to training, playing experience and skill level, were determinants of perceptual ability when recognising others from brief video displays of movement. No significance difference was found for each variable, even though Loula, Prassad, Harber, and Shiffrar $(2005)^{22}$ found that familiarity with the person observed was critical for accurate observation, this was not corroborated in the current study. Moreover, Weast et al (2014) ${ }^{19}$ showed that expertise plays a significant role in this perceptual ability despite the experience of current participants not following this trend. A possible explanation can be attributed to the expertise reversal effect whereby learning techniques that are useful for novices are not as effective for experts due to the management of cognitive load processes. ${ }^{23}$ While cognitive load was not measured here some participants stated they engaged consciously in the decision process in a more deliberate manner compared to before the study, this may be a matter for future studies to consider.

\section{CONCLUSION}

The current study investigated the teammate discrimination abilities of female water polo players during an intervention study. Unlike previous studies participants were not able to perform this task at a level significantly above chance at any testing point. Moreover, video training did not improve this ability despite previous studies suggesting the efficacy of video as a possible training method for biological motion. This may have been affected by the study limitations that include; duration of the training footage, quality of the image (frame rate), lack of representative design between test and training sequences and a small sample size. Despite these limitations, the findings provide a sound foundation for further training studies within the biological motion and sport domain with an emphasis on perceptual training for teammate recognition.

\section{REFERENCES}

1. Steel KA, Adams RD, Canning CG. Identifying swimmers as water-polo or swim teammates from visual displays of less than one second. Journal of Sports Sciences.

2007: 25(11), 1251-1258. doi: 10.1080/02640410601021721

2. Robbins R, \& McKone $E$. No face-like processing for objects-of-expertise in three behavioural tasks. Cognition, 2007: 103(1), 34-79.

3. Steel KA, Ellem E, \& Baxter D. The application of biological motion research: Biometrics, sport, and the military. Psychonomic Bulletin and Review, 2015: 22(1), 78-87. 
Brazilian Journal of Motor Behavior

\section{Research Article}

4. Richardson MJ, \& Johnston L. Person recognition from dynamic events: The kinematic specification of individual identity walking style. Journal of Nonverbal Behavior, 2006: 29(1), 25-44.

5. Stienen BMC, \& de Gelder B. Fear detection and visual awareness in perceiving bodily expressions. Emotion, 2011: 11(5), 1182-1189. doi: 10.1037/a0024032

6. Johansson $\mathrm{G}$. Visual perception of biological motion and a model for its analysis. Perception and Psychophysics, 1973: 14(2), 201-211.

7. Kozlowski LT, \& Cutting JE. Recognising the gender of walkers from point-lights mounted on ankles: Some second thoughts. Perception and Psychophysics, 1978: 23(5), 459.

8. Cutting JE, \& Kozlowski LT. Recognising friends by their walk; Gait perception without familiarity cues. Bulletin of the Psychonomic Society, 1977: 9(5) 353-356.

9. Dittrich WH. Action categories and the perception of biological motion. Perception, 1993: 22(1), 15-22. doi: 10.1068/p220015

10. Grossman E, Donnelly M, Price R, Pickens D, Morgan V, Neighbor G, Blake R. Brain areas involved in perception of biological motion. Journal of Cognitive Neuroscience. 2000;12(5):711-20.

11. Johnson HM. Biological motion: A perceptual life detector. Current Biology, 2006: 16(10) 376-377. doi: 10.1016/j.cub.2006.04.008

12. Bouchrika I, \& Nixon MS. People detection and recognition using gait for automated visual surveillance. IET Conference on Crime and Security, 2006: p. 576 - 581

13. Murray MP, Drought AB, \& Kory R C. Walking patterns of normal men. Journal of Bone and Joint Surgery, 1964: 46(2), 335-360.

14. Sayer JR, \& Mettford ML. High visibility safety apparel and nighttime conspicuity of pedestrians in work zones. Journal of Safety Research, 2004: 35(5), 537-546. doi: 10.1016/j.jsr.2004.08.007

15. Steel KA, Adams RD, Canning CG. Identifying runners as football teammates from 400 msec. video-clips. Perceptual and Motor Skills. 2006: 103(1), 901-911. doi: 10.2466/pms.103.3.901-911

16. Steel KA, Adams RD, Canning CG. Junior football player's classification of runners as their teammates from 400-msec video clips. Perceptual and Motor Skills. 2008: 107(1), 317322. doi: 10.2466/PMS.107.1.317-322

17. Appelbaum LG, Erickson G. Sports vision training: a review of the state-of-the-art in digital training techniques. International Review of Sport and Exercise Psychology. 2018;11(1):160-89.

18. McNicol D. A primer of signal detection theory: Psychology Press. 2005.

19. Weast JA, Walton A, Chandler B C, Shockley K, \& Riley M A. Essential kinematic information, athletic experience, and affordance perception for others. Psychonomic bulletin \& review, 2014: 21(3), 823-829. 
20. Pinder RA, Davids K, Renshaw I, Araújo D. Representative learning design and functionality of research and practice in sport. Journal of Sport and Exercise Psychology, 2011; 33(1):146-55.

21. Zhu FF, Poolton JM, Wilson MR, Maxwell JP, \& Masters RSW. Neural co-activation as a yardstick of implicit motor learning and the propensity for conscious control of movement. Biological Psychology, 2011: 87(1), 66-73.

22. Loula F, Prasad S, Harbe, K, \& Shiffrar M. Recognizing people from their movement. Journal of Experimental Psychology: Human Perception and Performance, 2005: 31(1), 210.

23. Kalyuga $S$, Ayres $P$, Chandler $P, \&$ Sweller J. The expertise reversal effect. Educational Psychologist, 2003: 38(1), 23-31.

\section{ACKNOWLEDGEMENT}

The author would like to acknowledge the assistance of Dr Roger Adams and Associate Professor Colleen Canning for their assistance and guidance in the conception, and initial data analysis for this study. And also Mr Paul Fahey, who assisted with final data analysis.

\footnotetext{
Citation: Steel, KA. Non-Support for Trainability of Teammate Recognition Based on Movement Perception?. BJMB. 2019: 13(1): 32-41.

Editor: Dr Fabio Augusto Barbieri - São Paulo State University (UNESP), Bauru, SP, Brazil; Dr José Angelo Barela São Paulo State University (UNESP), Rio Claro, SP, Brazil; Dr Natalia Madalena Rinaldi - Federal University of Espírito Santo (UFES), Vitória, ES, Brazil.

Copyright: (C) 2019 Steel and BJMB. This is an open-access article distributed under the terms of the Creative Commons Attribution-NonCommercial-NoDerivatives 4.0 International License which permits unrestricted use, distribution, and reproduction in any medium, provided the original author and source are credited.

Funding: There was no funding for this study.

Competing interests: The authors have declared that no competing interests exist.

DOl: https://doi.org/10.20338/bimb.v13i1.120
}

\begin{tabular}{l|l|l|l|}
\hline Steel & 2019 & VOL.13 & N.1 \\
\end{tabular}

